\title{
Sequential Carotenoids Extraction and Biodiesel Production from Rhodosporidium toruloides NCYC 921 Biomass
}

\author{
Paula C. Passarinho ${ }^{1} \cdot$ Bruno Oliveira $^{1} \cdot$ Carla Dias $^{1} \cdot$ Marta Teles $^{1} \cdot$ Alberto Reis $^{1} \cdot$ Teresa Lopes da Silva $^{1}$ (i)
}

Received: 15 May 2018 / Accepted: 21 October 2018

(c) Springer Nature B.V. 2018

\begin{abstract}
A new process for co-extraction and separation of fatty acids and carotenoids from Rhodosporidium toruloides NCYC 921 biomass in order to achieve full exploitation of the yeast lipidic fraction is described. A saponification of the wet yeast biomass was performed using a potassium hydroxide solution $(1.1 \mathrm{M})$ in ethanol $96 \%$, at $65^{\circ} \mathrm{C}$ for $180 \mathrm{~min}$. In the carotenoid extraction step, a biphasic system with an organic:aqueous phases ratio of $0.49 \mathrm{~mL} / \mathrm{mL}$ and a water content of $18.9 \%$ (w/w) was used. In the presence of an acid catalyst, the fatty acid fraction was esterified into fatty acids ethyl esters. The yeast biomass downstream processing allowed reaching a fatty acid and total carotenoids recovery yields of $91.0 \%$ and $85.2 \%$, respectively. The process reported here takes advantage of various components of the yeast biomass, therefore maximizing the value derived from the biomass feedstock, with a minimal environmental impact within the frame of circular bioeconomy.
\end{abstract}

Keywords Rhodosporidium toruloides $\cdot$ Saponification $\cdot$ Carotenoids $\cdot$ Biodiesel $\cdot$ Downstream processing $\cdot$ Biorefinery $\cdot$ Yeast

\section{Statement of Novelty}

The present study presents a novel, inexpensive and environmentally friendly process involving the simultaneous extraction of carotenoids and saponifiable lipids from the same $R$. toruloides NCYC 921 biomass sample, using a sequential process of saponification, followed by liquid-liquid extraction and esterification of the fatty acids, resulting in the coproduction of fatty acids ethyl esters (FAEE; biodiesel) and carotenoids, never reported before.

The process here discussed takes advantage of the various yeast biomass components, producing biodiesel, carotenoids and, potentially, other byproducts along the process. The inclusion of all these operations will greatly improve the economics of the overall process, as the high-value added products obtained in the process will economically sustain the microbial biodiesel production.

Teresa Lopes da Silva

teresa.lopessilva@lneg.pt

1 Unidade de Bioenergia, Laboratório Nacional de Energia e Geologia, I.P., Estrada do Paço do Lumiar, 22, 1649-038 Lisbon, Portugal

\section{Introduction}

Due to the increasing difficulty in accessing and extracting fossil fuels, their expected medium and long-term scarcity, and the environmental problems caused by their use (increase of greenhouse gases in the atmosphere resulting in climate changes), the scientific community has been searching for renewable energy sources whose production and use are economically and environmentally sustainable.

Biodiesel is a renewable, non-toxic biofuel, which, when used in the transport sector, emits less pollutant gases than fossil diesel. Transesterification is the conventional process for biodiesel production from oils and fats resulting in general in methyl fatty acid esters and glycerol. No other high value added lipidic products are produced in this process.

Also, the use of arable soils for vegetable oleaginous crops (usually corn, soybean, rape, sunflower) that are processed into biodiesel, instead of being used in the food industry, has resulted in higher prices for these products, raising public controversy all over the world [1]. Therefore, nowadays, new sources of oils and fats for the production of biodiesel are being searched and oleaginous microorganisms are a very good feedstock alternative. Autotrophic oleaginous microalgae have been suggested as possible sources of oil for biodiesel production [2]. However, the use of 